\title{
Supporting Information: Effect of Phase Transition on the Thermal Transport in Isoreticular DUT Materials
}

\author{
Penghua Ying, Jin Zhang, ${ }^{*}$ and Zheng Zhong* \\ School of Science, Harbin Institute of Technology, Shenzhen 518055, PR China \\ E-mail: jinzhang@hit.edu.cn; zhongzheng@hit.edu.cn
}

\section{Pressure-versus-volume equations of state calculation}

The pressure-versus-volume equations of state $(P-V$ EOS $)$ calculations are adopted in our work to determine the phase transition and the np phase of isoreticular DUT crystals. This method has been widely used to provide thermodynamic insight into stimuli-responsive behaviour of SPCs. ${ }^{1-10}$ The $P-V E O S$ is constructed by discrete points. The profile of pressure is calculated as a function of unit-cell volume. Based on the obtained $P-V$ EOS, the free energy $F$ can be calculated by the following thermodynamics integration ${ }^{11}$

$$
F(V)=F\left(V_{0}\right)+\int_{V_{0}}^{V} P\left(V^{\prime}\right) d V^{\prime}
$$

Here, $V_{0}$ is the volume of reference configuration, which is taken as the base point of integration. We set the lp phase as the reference configuration in this paper. The obtained free energy-versus-volume curves is obtained by applying the ninth-order polynomial fitting to the discrete points. 
In our calculations, the $P-V$ EOS was obtained by performing MD simulations to a series of volume points within the range of interest. Specifically, the crystal length of optimized DUT materials was first increased by $0.5 \AA$ to obtain complete EOS picture, and then decreased by $0.2 \AA$ in each loop until enough EOS information has been collected. In each loop, the MD simulation was implemented in NVT ensemble. Here, the required pressure was calculated by averaging the simulation results of $100 \mathrm{ps}$ after obtaining the equilibrium state.

\section{Calculation of elastic constants}

The strain-fluctuation method ${ }^{12}$ was adopted here to obtain the elastic constants of lp and np structures of DUT crystals, which is based on the following analysis of the fluctuations of unit cell vectors:

$$
C_{i j k l}^{-1}=\frac{V}{k_{B} T}\left(\left\langle\varepsilon_{i j} \varepsilon_{k l}\right\rangle-\left\langle\varepsilon_{i j}\right\rangle\left\langle\varepsilon_{k l}\right\rangle\right),
$$

where $k_{B}$ is Boltzmann's constant, $T$ is system temperature, $V$ is the volume of the simulation

box, $C_{i j k l}^{-1}$ denotes the compliance tensor that is the inverse of the stiffness tensor, and symbol \langle\rangle represents the average of sampling points in the entire simulation process. The NsT ensemble was selected to obtain the sampling points. The sampling time in each MD simulation is $5 \mathrm{~ns}$, followed by $1 \mathrm{~ns}$ relaxation to reach an equilibrium state. The unit cell vector is output every $10 \mathrm{fs}$, corresponding to 500,000 discrete points used to calculate the elastic stiffness tensor. Due to translational and rotational symmetries, the number of independent components of $C$ reduces from 81 to 21. In Voigt notation the fourth order elastic stiffness tensor $C_{i j k l}$ can be represented by a matrix in a 6 -dimensional vector space $C_{i j}$ (see Marmier's work ${ }^{13}$ for the specific conversion relationship). The elastic stiffness matrix $C_{i j}$ is then defined as the second derivative of elastic energy density $E$ to the strains $\varepsilon_{i}$ and $\varepsilon_{j}$ :

$$
C_{i j}=\frac{\partial^{2} E}{\partial \varepsilon_{i} \partial \varepsilon_{j}}
$$


For highly symmetric cubic isoreticular DUT materials investigated in our study, there are only three independent elastic constants $\mathrm{C}_{11}, \mathrm{C}_{12}$, and $\mathrm{C}_{44}$, which are, respectively, the modulus of axial compression, dilation on compression, and shearing. The bulk modulus $K$, shear modulus $G$, and Young's modulus $E$ of cubic crystals in Voigt scheme are given by:

$$
K=\frac{\mathrm{C}_{11}+2 \mathrm{C}_{12}}{3}, G=\frac{\mathrm{C}_{11}-\mathrm{C}_{12}+3 \mathrm{C}_{44}}{3}, E=\left(\frac{1}{3 G}+\frac{1}{9 K}\right)^{-1} .
$$

The three-dimensional spatial dependence Young's modulus was obtained with the aid of the coordinate transformation introduced in Marmier et al.'s work. ${ }^{13}$

\section{Figures}
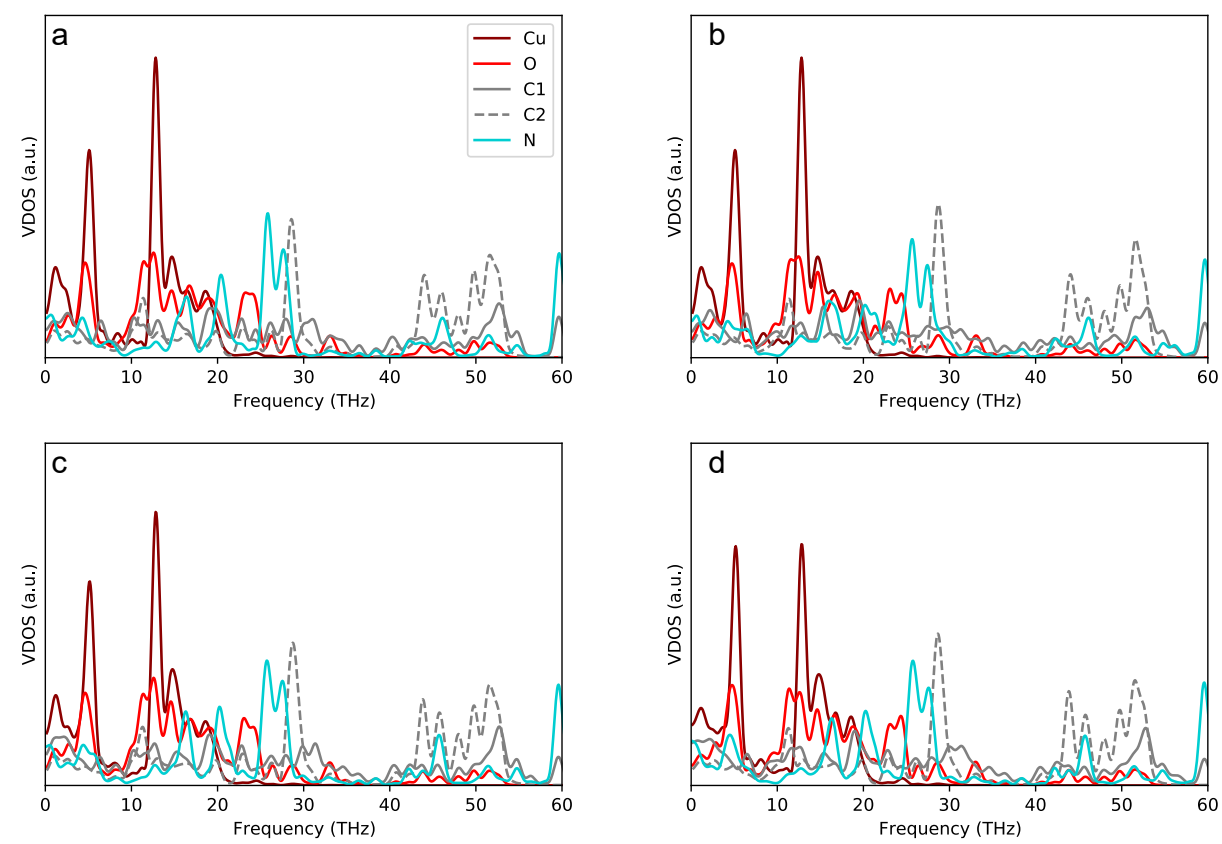

Figure S1: VDOS distribution of the framework atoms of isoreticular DUT crystals: (a) DUT-48, (b) DUT-47, (c) DUT-50, and (d) DUT-151. 

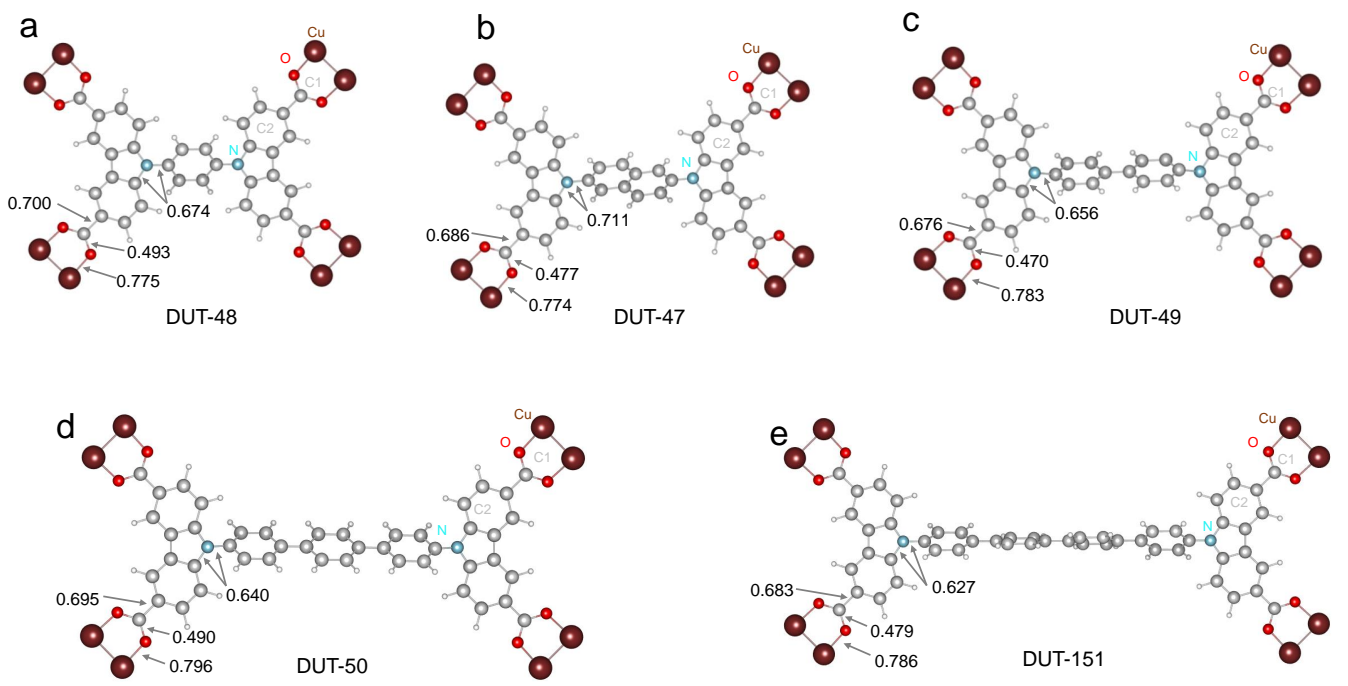

Figure S2: The similarity index calculated at 0-60 THz of atomic bonds in isoreticular DUT crystals: (a) DUT-48, (b) DUT-47, (c) DUT-49, (d) DUT-50, and (e) DUT-151.

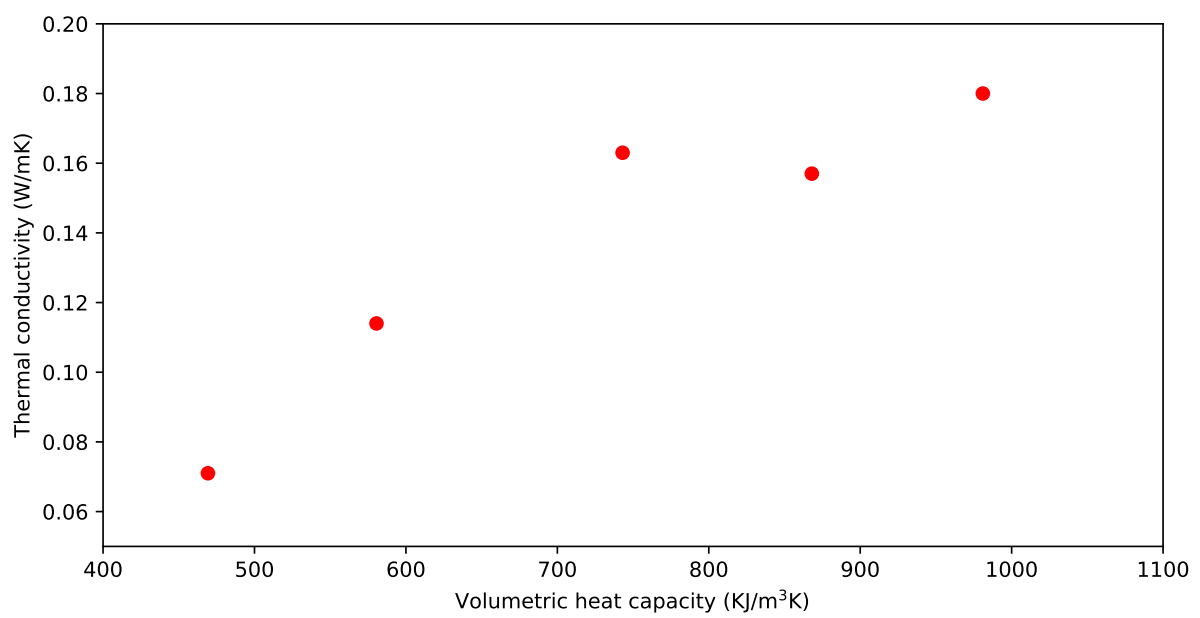

Figure S3: The thermal conductivity of lp phase of DUT materials as a function of their volumetric heat capacity. 

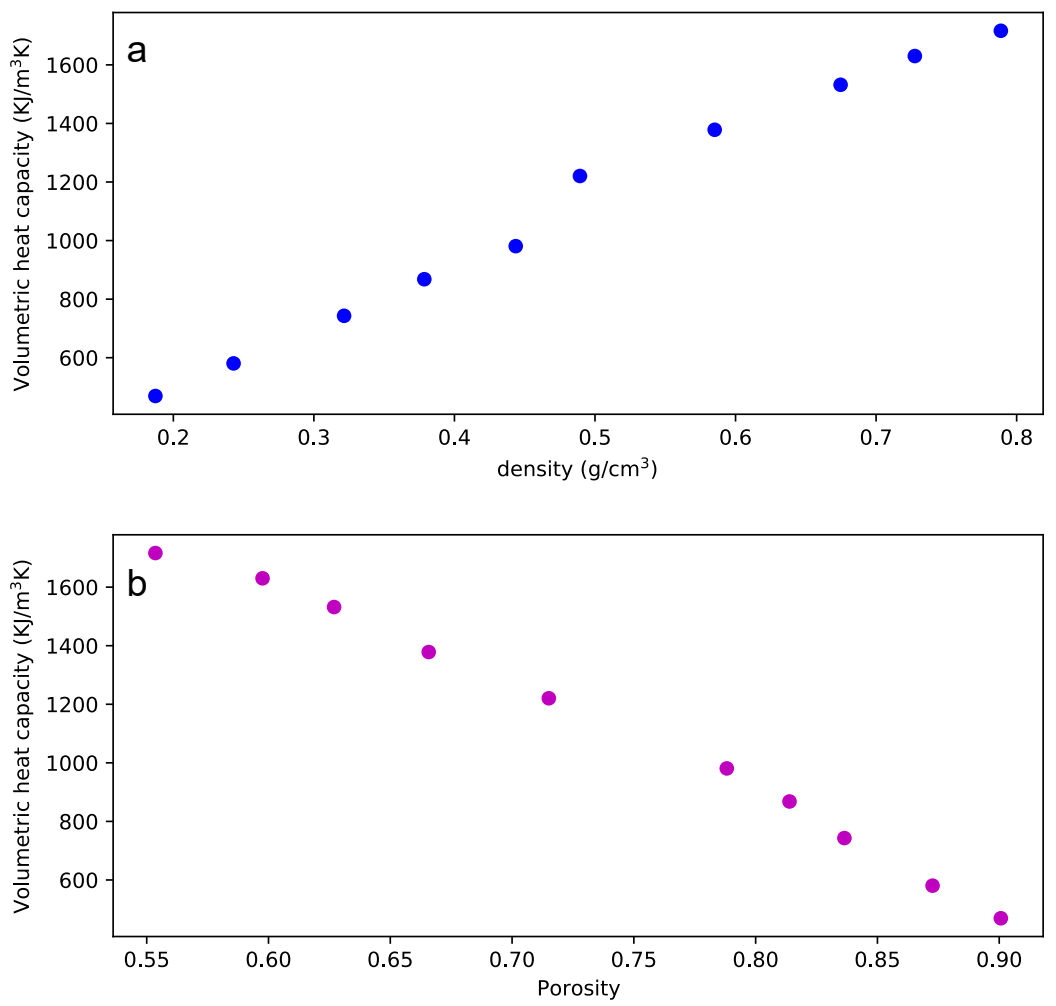

Figure S4: (a) The volumetric heat capacity of DUT materials as a function of their (a) density and (b) porosity. Here, both lp and np structures are considered.

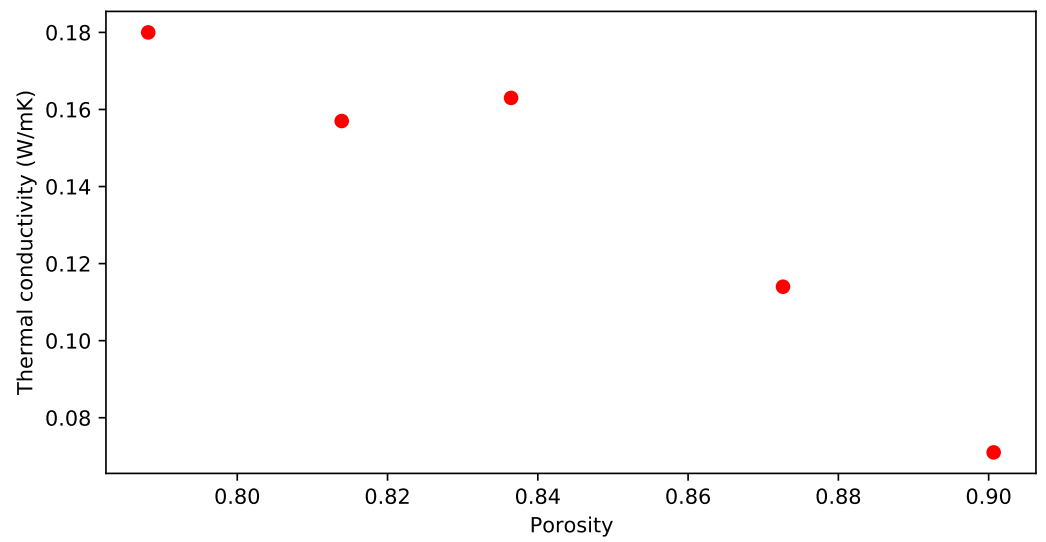

Figure S5: The thermal conductivity of DUT crystals as a function of their porosity. 

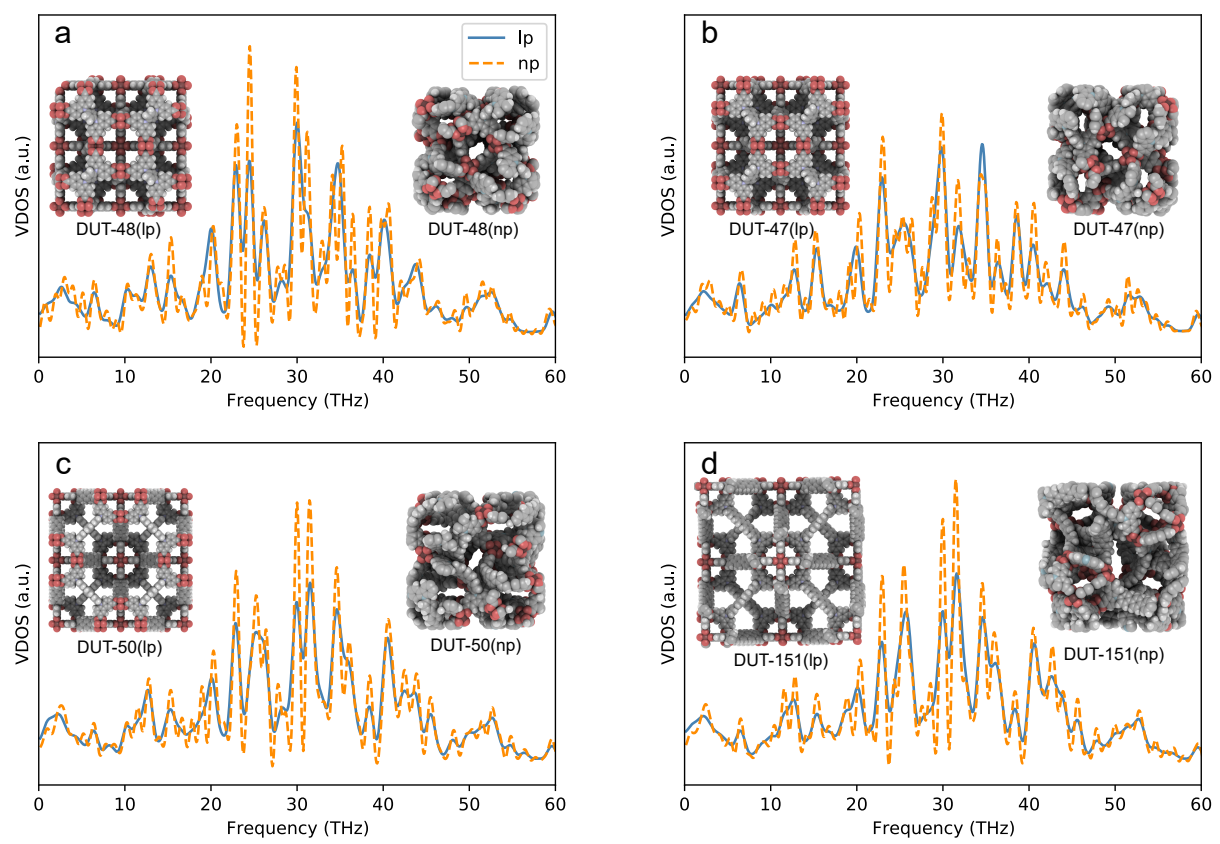

Figure S6: A comparison of the framework VDOS of lp and np phases of DUT crystals: (a) DUT-48, (b) DUT-47, (c) DUT-50, and (d) DUT-151.
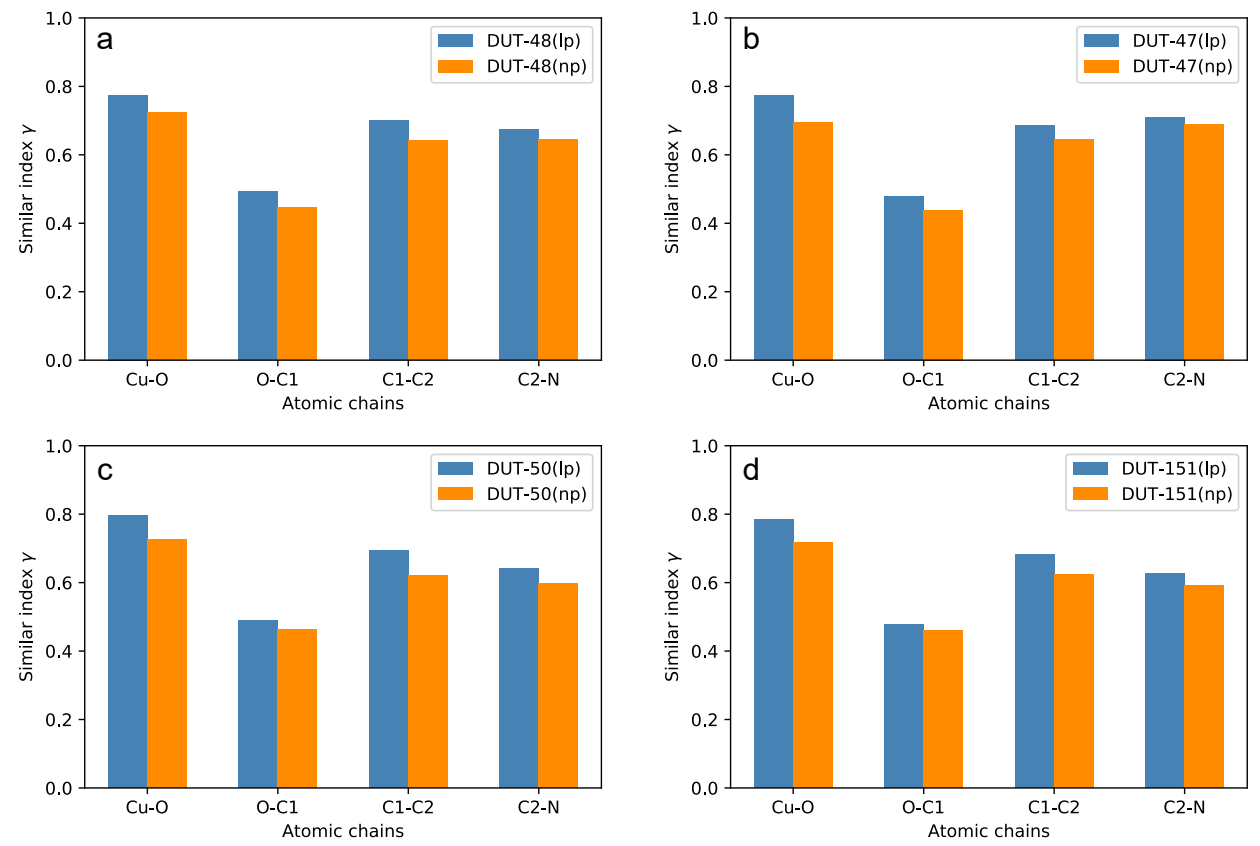

Figure S7: The similarity index $\gamma$ of atomic VDOS overlap calculated at 0-60 THz for the lp and np phases of DUT crystals: (a) DUT-48, (b) DUT-47, (c) DUT-50, and (d) DUT-151. 


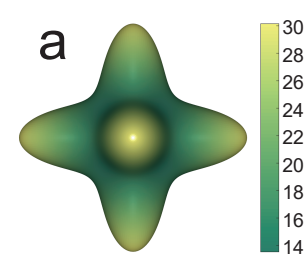

DUT-48(Ip)

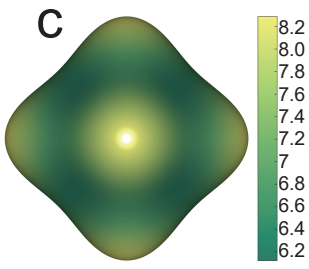

DUT-50(Ip)

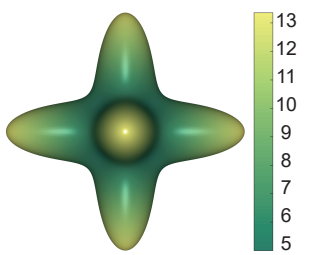

DUT-48(np)

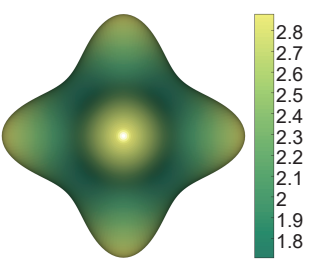

DUT-50(np)

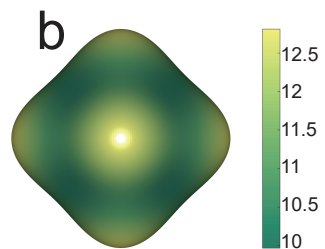

DUT-47(Ip)

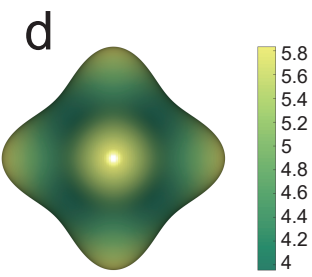

DUT-151(Ip)

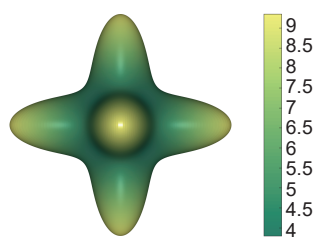

DUT-47(np)

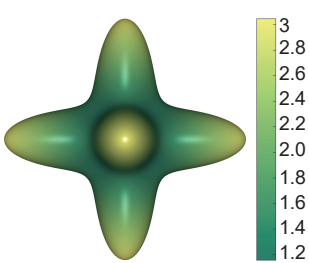

DUT-151(np)

Figure S8: Spatial dependence of Young's modulus of lp and np phases of (a) DUT-48, (b) DUT-47, (C) DUT-50, and (d) DUT-151. All results are in the unit of GPa.

\section{Tables}

Table S1: A comparison of volumetric heat capacity of DUT crystals obtained by directly derivative method and energy fluctuations method ${ }^{a}$

\begin{tabular}{ccc} 
DUT Crystals & $C_{V}^{d}$ & $C_{V}^{f}$ \\
\hline DUT-48(lp) & 981 & 1001 \\
DUT-48(np) & 1716 & 1765 \\
DUT-47(lp) & 868 & 865 \\
DUT-47(np) & 1630 & 1642 \\
DUT-49(lp) & 743 & 742 \\
DUT-49(np) & 1532 & 1539 \\
DUT-50(lp) & 581 & 593 \\
DUT-50(np) & 1378 & 1406 \\
DUT-151(lp) & 469 & 474 \\
DUT-151(np) & 1221 & 1255
\end{tabular}

${ }^{a} C_{V}^{d}$ and $C_{V}^{f}$ denote the volumetric heat capacity obtained by derivative method (i.e., directly calculated the heat capacity based on change in the internal energy with temperature, see Equation 3) and energy fluctuations method (see Equation 4), respectively. All results are in the unit of $\mathrm{KJ} /\left(\mathrm{m}^{3} \mathrm{~K}\right)$. 
Table S2: Elastic Constants and Mechanical Properties of DUT crystals $^{a}$

\begin{tabular}{ccccccc} 
DUT Crystals & $\mathrm{C}_{11}$ & $\mathrm{C}_{12}$ & $\mathrm{C}_{44}$ & $E$ & $G$ & $B$ \\
\hline DUT-48(lp) & 30.6 & 2.7 & 5.2 & 21.0 & 8.7 & 12.0 \\
DUT-48(np) & 14.2 & 2.6 & 1.8 & 8.7 & 3.4 & 6.5 \\
DUT-47(lp) & 15.0 & 4.7 & 3.8 & 11.1 & 4.4 & 8.1 \\
DUT-47(np) & 12.5 & 5.2 & 1.3 & 6.1 & 2.2 & 7.6 \\
DUT-49(lp) & 15.4 & 3.5 & 3.2 & 10.8 & 4.3 & 7.5 \\
DUT-49(np) & 9.7 & 3.5 & 0.9 & 4.9 & 1.8 & 5.6 \\
DUT-50(lp) & 9.8 & 3.1 & 2.3 & 7.0 & 2.7 & 5.4 \\
DUT-50(np) & 5.1 & 3.0 & 0.6 & 2.1 & 0.7 & 3.7 \\
DUT-151(lp) & 7.1 & 2.4 & 1.5 & 4.7 & 1.8 & 4.0 \\
DUT-151(np) & 5.5 & 3.3 & 0.4 & 1.9 & 0.7 & 4.0
\end{tabular}

${ }^{a} E, G$, and $B$ denote the Young's modulus, shear modulus and bulk modulus in Voigt scheme, ${ }^{13}$ respectively. All results are in the unit of GPa. 


\section{References}

[S1] Vanduyfhuys, L.; Rogge, S.; Wieme, J.; Vandenbrande, S.; Maurin, G.; Waroquier, M.; Van Speybroeck, V. Thermodynamic insight into stimuli-responsive behaviour of soft porous crystals. Nature communications 2018, 9, 1-9.

[S2] Rogge, S. M.; Waroquier, M.; Van Speybroeck, V. Unraveling the thermodynamic criteria for size-dependent spontaneous phase separation in soft porous crystals. Nature communications 2019, 10, 1-10.

[S3] Wieme, J.; Lejaeghere, K.; Kresse, G.; Van Speybroeck, V. Tuning the balance between dispersion and entropy to design temperature-responsive flexible metal-organic frameworks. Nature communications 2018, 9, 1-10.

[S4] Demuynck, R.; Rogge, S. M.; Vanduyfhuys, L.; Wieme, J.; Waroquier, M.; Van Speybroeck, V. Efficient construction of free energy profiles of breathing metal-organic frameworks using advanced molecular dynamics simulations. Journal of chemical theory and computation 2017, 13, 5861-5873.

[S5] Keupp, J.; Schmid, R. Molecular dynamics simulations of the "breathing" phase transformation of MOF nanocrystallites. Advanced Theory and Simulations 2019, 2, 1900117.

[S6] Vervoorts, P.; Keupp, J.; Schneemann, A.; Hobday, C. L.; Daisenberger, D.; Fischer, R. A.; Schmid, R.; Kieslich, G. Configurational Entropy Driven High-Pressure Behaviour of a Flexible Metal-Organic Framework (MOF). Angewandte Chemie 2020,

[S7] Evans, J. D.; Bocquet, L.; Coudert, F.-X. Origins of negative gas adsorption. Chem 2016, 1, 873-886.

[S8] Krause, S.; Evans, J. D.; Bon, V.; Senkovska, I.; Iacomi, P.; Kolbe, F.; Ehrling, S.; Troschke, E.; Getzschmann, J.; Többens, D. M., et al. Towards general network ar- 
chitecture design criteria for negative gas adsorption transitions in ultraporous frameworks. Nature communications 2019, 10, 1-12.

[S9] Ying, P.; Zhang, J.; Zhong, Z. Pressure-induced phase transition of isoreticular MOFs: Mechanical instability due to ligand buckling. Microporous and Mesoporous Materials 2020, 110765.

[S10] Rogge, S. M.; Waroquier, M.; Van Speybroeck, V. Reliably modeling the mechanical stability of rigid and flexible metal-organic frameworks. Accounts of chemical research 2018, 51, 138-148.

[S11] Kirkwood, J. G. Statistical mechanics of fluid mixtures. The Journal of chemical physics 1935, 3, 300-313.

[S12] Parrinello, M.; Rahman, A. Strain fluctuations and elastic constants. The Journal of Chemical Physics 1982, 76, 2662-2666.

[S13] Marmier, A.; Lethbridge, Z. A.; Walton, R. I.; Smith, C. W.; Parker, S. C.; Evans, K. E. ElAM: A computer program for the analysis and representation of anisotropic elastic properties. Computer Physics Communications 2010, 181, 2102-2115. 